\title{
Nota
}

\section{PENETRÔMETRO DE IMPACTO STOLF - PROGRAMA COMPUTACIONAL DE DADOS EM EXCEL-VBA ${ }^{(1)}$}

\author{
Rubismar Stolf ${ }^{(2)}$, Jorge Hiroshi Murakami ${ }^{(3)}$, Caetano Brugnaro ${ }^{(4)}$, Luiz Gabriel Silva ${ }^{(5)}$, \\ Luiz Carlos Ferreira da Silva ${ }^{(2)} \&$ Luiz Antonio Correia Margarido ${ }^{(4)}$
}

\section{RESUMO}

Há dois principais tipos de penetrômetro, o estático e o dinâmico. No primeiro, uma haste com uma ponta cônica é introduzida contínua e lentamente (quase estático), registrando-se concomitantemente a força de reação que é igual à resistência do solo. No segundo, utiliza-se a mesma haste. Contudo, esta é introduzida por meio da promoção de uma massa de impacto em queda livre. Dessa forma, a teoria pode ser tratada pela dinâmica newtoniana para obtenção da resistência. $O$ objetivo do programa é proporcionar uma ferramenta rápida, facilitadora da computação de dados de resistência do solo, para esse último penetrômetro, resultando tabelas e gráficos já no formato científico. Desenvolvido em linguagem de programação Visual Basic Aplication (VBA), escolheu-se o aplicativo Excel como interface com o usuário pela sua popularidade. É constituído por quatro planilhas, duasauxiliares, sendo duas delas essenciais, Plan1 e 2: Plan1 - dados de entrada (número de impactos e profundidade). Concomitante à digitação, a tabela de resistência é confeccionada com o respectivo gráfico, podendo tabelar 40 perfis. Plan2 - cumpre função especial de equalizar, ou seja, padronizar a profundidade em camadas constantes, permitindo unificar todos os perfis em uma única tabela. Para tal, escolhe-se uma espessura de camada (exemplo: 5 $\mathrm{cm}$ ); em seguida, o programa coleta os dados de resistência (MPa) da Plan1 e interpola os valores para a profundidade de $5 \mathrm{em} 5 \mathrm{~cm}$. Após realizar esse procedimento para todas as tabelas da Plan1, o programa gera, na Plan2, uma única tabela com todos os perfis, a média geral e os respectivos gráficos. É possível selecionar os perfis; por exemplo, apenas os medidos na linha ou entrelinha de plantio e eliminar perfis a critério do usuário. Como objetivo complementar, descreve-se a evolução do projeto "Penetrômetro de impacto",

(1) Software gratuito: <http://www.cca.ufscar.br/drnpa/hprubismar_ARTIGOS/100._Penetrometro(Stolf,R).xls>. Trabalho apresentado no XLII Congresso Brasileiro de Engenharia Agrícola - CONBEA 2013. Recebido para publicação em 23 de setembro de 2013 e aprovado em 27 de março de 2014.

(2) Professor Associado, Departamento de Recursos Naturais e Proteção Ambiental, Centro de Ciências Agrárias, Universidade Federal de São Carlos - UFSCar. Via Anhanguera, km 174. CEP 13600-970 Araras (SP). E-mail: rubismar@cca.ufscar.br

(3) Engenheiro Mecânico, Departamento de Desenvolvimento de Produto, Kamaq - Máquinas e Implementos Agrícolas. Via Industrial, 550. CEP 13602-030 Araras (SP). E-mail: jorgehm@kamaq.com.br

(4) Professor Associado, Departamento de Tecnologia Agroindustrial e Sócio-Economia Rural, UFSCar. E-mail: brugnaro@cca.ufscar.br,marga@cca.ufscar.br

(5) Discente de Engenharia Agronômica, UFSCar. E-mail: lgs346900@gmail.com 


\begin{abstract}
iniciado em 1982, cuja técnica passou a ser adotada no meio científico, após a publicação da teoria nesta revista em 1991. Apresenta-se também levantamento no meio científico nos últimos três anos (2010-2012), propiciando uma visão das principais aplicações na atualidade.
\end{abstract}

Termos de indexação: perfil de resistência mecânica do solo, transformação de dados, confecção automática de tabelas e gráficos, uniformização da profundidade.

\title{
SUMMARY: STOLF IMPACT PENETROMETER - COMPUTER DATA PROGRAM IN EXCEL-VBA
}

\begin{abstract}
There are two main types of penetrometers. In the static one, a rod with a conical tip is introduced continuously and gradually (nearly statically), simultaneously registering the reaction force, which is equal to the soil resistance. The dynamic penetrometer uses the same rod. However, it is introduced through bringing about an impact mass in freefall. Thus, the theory can be treated by Newtonian dynamics so as to obtain dynamic resistance. The aim of the software is to provide a quick tool, facilitating the computation of soil resistance data for the latter type of penetrometer, yielding tables and graphs already in scientific format. Developed in VBA (Visual Basic for Applications) programming language, it uses Excel as the user interface due to its popularity. It basically consists of two worksheets, Sheet 1 and 2: Sheet 1 - this is for data entry (number of impacts and depth). Concomitant with typing in data, the resistance table is prepared with the respective graph, accepting up to 40 profiles. Sheet 2 - it has a special equalizing function, i.e., it standardizes depth in constant layers, unifying all profiles in a single table. For that purpose, after selection of a layer thickness (e.g., $5 \mathrm{~cm})$, the program collects the resistance data $(\mathrm{MPa})$ from Sheet 1 and interpolates the values for depths of every $5 \mathrm{~cm}$. After performing this procedure for all the tables in Sheet 1, the program generates, in Sheet 2, a single table with all the profiles and the overall average, with their graphs. The user can select the profiles - for example, only those measured in the plant row or interrow - or even exclude profiles. As a supplement aim, this study describes the evolution of the "Impact penetrometer" project, which began in 1982, and whose technique came to be adopted in the scientific community after publication of the theory in this journal in 1991. A survey of the scientific community over the past three years (2010-2012) is also presented, providing a view of the main current applications.
\end{abstract}

Index terms: mechanical soil resistance profile, data transformation, automatic preparation of tables and graphs, standardization of depth.

\section{INTRODUÇÃO}

O penetrômetro de impacto Stolf é um aparelho de medida da resistência do solo, do tipo dinâmico, cuja penetração ocorre por impacto. Lançado em 1982, tornou-se popular entre os agricultores por meio de publicações técnicas que abordavam aspectos práticos, ou seja, características do equipamento e da operação (Stolf et al., 1983) e outras exemplificando o uso em cana-de-açúcar (Stolf \& Faganello, 1983) e em pomares de laranja (Stolf, 1987). Nos trabalhos iniciais, adotou-se a unidade prática de resistência do solo, impactos/dm, comum em engenharia civil. A teoria para transformação da resistência em $\mathrm{MPa}$ foi apresentada inicialmente em congresso (Stolf, 1990a,b). Difundida em revista de alto impacto (Stolf, 1991a; Stolf et al., 1998; 2005), possibilitou tornar o aparelho conhecido no meio científico. A consolidação do penetrômetro de impacto Stolf nesse meio pode ser medida pelo seu emprego na atualidade. Verificase sua utilização em diversos estudos,como: mapeamento da resistência do solo, avaliação da compactação e de controle de tráfego, variabilidade espacial de propriedades do solo, manejo de lavouras, pastagens e florestas, recuperação de áreas degradadas, indicador de qualidade do solo, sedimentos em áreas ripárias, conforme os seguintes artigos científicos publicados, no período de 2010 a 2012 (Camargo et al., 2010; Cavallini et al., 2010; Correa \& Bento, 2010; Ferreira et al., 2010; Guedes Filho et al., 2010; Lima et al., 2010; Machado et al., 2010; Martins et al., 2010; Medeiros et al., 2010; Mestas et al., 2010; Oliveira et al., 2010; Pedron et al., 2010; Portugal et al., 2010; Ramos et al., 2010; Roboredo et al., 2010; Roque et al., 2010; Silva et al., 2010; Silva Filho et al., 2010; Silveira et al., 2010; Siqueira et al., 2010; Souza et al., 2010; Tavares Filho et al., 2010; Cardoso et al., 2011; Cortez et al., 2011; Costa et al., 2011; Iarema et al., 2011; Manfré et al., 2011; Marasca, 2011; Ramos et al., 2011; Silva et al., 2011; Theodoro et al., 2011; Tieppo et al., 2011; Torres et al., 2011; Almeida et al., 2012; Carvalho et al., 2012; Castagnara et al., 2012; Cobra et al., 2012; Cunha et al., 2012; Debiasi et al., 2012; Ecco et al., 2012; Effgen et al., 2012; Freitas et al., 2012; Grego et al., 2012; Homma et al., 2012; Molin et al., 2012; Montanari et 
al., 2012; Moura et al., 2012; Silva et al., 2012; Tavares Filho et al., 2012; e Wendling et al., 2012).

Recentemente, foram estabelecidos dois projetos de desenvolvimento de tecnologia facilitadora do uso do penetrômetro de impacto. O primeiro, já concluído, refere-se à introdução de uma régua facilitadora de leitura de profundidade (Stolf et al., 2012); e o segundo, o presente projeto, para solucionar dificuldades quanto à computação de dados, descritas a seguir.

A questão da computação de dados em penetrômetro de impacto: na utilização de penetrômetros de impacto, o operador não tem controle quanto à espessura da camada penetrada provocada pelo impacto. Portanto, não é possível gerar resultados de resistência em intervalos de profundidade constante, como nas camadas de 0-5; 5-10; e 10-15 cm. Esse aspecto dificulta a análise de resultados de resistência, de vários perfis, em camadas na mesma profundidade, e estudos de variabilidade espacial. Para contornar essa dificuldade, criou-se um método manual de tabelamento descrito em Stolf et al. (1983). Em seguida, desenvolveu-se, em 1985, para o computador de mesa científico HP85, um programa de uso interno da instituição, pois não havia procedimento de divulgação (época anterior ao shareware). Em 1990, no início da era PC, o penetrômetro foi programado para uso em um projeto nos EUA. Contudo, esse modelo de computador, HP85 , entrou em desuso. Nesse segundo momento, foi necessário desenvolver rapidamente um programa computacional (Stolf, 1991b), utilizando o sistema operacional DOS na linguagem Quick Basicpara uso no citado projeto (Stolf et al., 1998). Apesar de cumprir a função de equalizar os intervalos de profundidade das medidas de resistência, esse outro programa não foi considerado um produto acabado, não se promovendo a divulgação. Mesmo assim, é possível encontrar na literatura alguma citação de uso mais recente (Araujo et al., 2004; Homma, 2005; Iarema et al., 2011), evidenciando, pelo contraste da antiguidade do programa (1991), a necessidade de se desenvolver um programa computacional moderno para esse tipo de penetrômetro.

Dessa forma, estabeleceu-se, como objetivo deste trabalho, o desenvolvimento de um programa que permita a realização das seguintes atividades computacionais:

a. Confecção automática da tabela e do gráfico de resistência na medida em que se dá entrada aos dados de número de impactos e profundidade;

b. Equalização da profundidade em camadas constantes, segundo a preferência do usuário, unificando todos os perfis em uma única tabela, já no formato científico. Além disso, prevê-se a inclusão de uma coluna com o perfil médio de resistência acompanhada do respectivo gráfico;

c. Possibilidadede se trabalhar separadamente, por exemplo: linha $\times$ entrelinha; mata $\times$ área agrícola; $\mathrm{e}$ plantio direto $\times$ convencional, ao entrar uma série de perfis; e d. Adoção de uma planilha popular conhecida como interface com o usuário.

\section{MATERIAL E MÉTODOS}

A interface com o usuário escolhida foi a planilha Excel; e a linguagem de programação adotada, a Visual Basic Aplication (VBA), ambas da Microsoft.

\section{Conversão do número de impactos em resistência $(R)$}

O programa apresentará a resistência do solo (R) nas unidades: impactos $/ \mathrm{dm}, \mathrm{kgf} / \mathrm{cm}^{2}$ e $\mathrm{MPa}$. A equação para cálculo da resistência em razão da penetração por impacto foi desenvolvida em Stolf (1991a) e reapresentada em Stolf et al. (1998; 2005):

Unidade prática: $\mathrm{N}($ Impactos $/ \mathrm{dm})=10 \times \mathrm{n}^{\mathrm{o}} \mathrm{de}$ impactos/penetração $(\mathrm{cm})$

Unidade técnica:

$$
\mathrm{R}\left(\mathrm{kgf} / \mathrm{cm}^{2}\right)=5,6+6,89 \times \mathrm{N}(\mathrm{impactos} / \mathrm{dm})
$$

Unidade utilizada em artigos científicos, $\mathrm{MPa}$, para $\mathrm{g}=10 \mathrm{~m} / \mathrm{s}^{2}$ :

$$
\mathrm{R}(\mathrm{MPa})=0,56+0,689 \times \mathrm{N}(\mathrm{impactos} / \mathrm{dm}),
$$

ou seja, $\mathrm{R}(\mathrm{MPa})=0,1 \mathrm{R}\left(\mathrm{kgf} / \mathrm{cm}^{2}\right)$.

Exemplificando: com dois impactos, a profundidade variou de 11 a $15 \mathrm{~cm}$.

$\mathrm{N}(\operatorname{Impactos} / \mathrm{dm})=10 \times 2 /(15-11)=5$, ou seja, resistência $=5$ impactos $/ \mathrm{dm}$;

$\mathrm{R}\left(\mathrm{kgf} / \mathrm{cm}^{2}\right)=5,6+6,89 \times 5=40,1$, ou seja, resistência $=40,1 \mathrm{kgf} / \mathrm{cm}^{2}$;

$\mathrm{R}(\mathrm{MPa})=0,1 \times 40,1=4,01$, ou seja, resistência $=$ 4,01 MPa.

\section{Método computacional}

$\mathrm{O}$ aspecto central do programa é transformar em intervalos constantes de profundidade os resultados de resistência do solo, pela impossibilidade de realizar leituras a intervalos constantes de profundidade. $\mathrm{O}$ processo adotado para tal é ilustrado no esquema da figura 1, cujos valores de resistência foram transformados para intervalos de $5 \mathrm{~cm}$, como exemplo. No programa, os intervalos de profundidade foram tomados como variável de forma que o usuário possa escolher outro intervalo, inclusive número fracionário.

\section{RESULTADOS E DISCUSSÃO}

Desenvolvido em linguagem VBA, adotou-se a planilha Excel para interface com usuário pela simplicidade. O programa Excel é constituído 


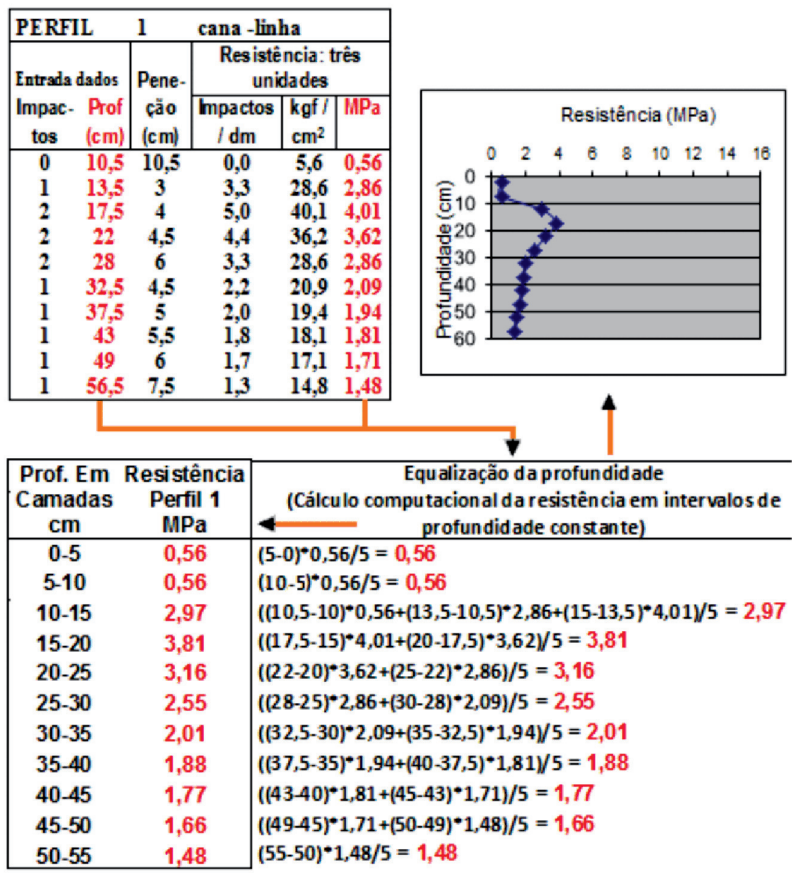

Figura 1. Esquema da amostragem de um perfil de solo até a profundidade de $56,5 \mathrm{~cm}$ e respectivas resistências e, abaixo, o processo da obtenção da resistência pela média ponderada para intervalos de profundidade de $5 \mathrm{em} 5 \mathrm{~cm}$.

basicamente de três planilhas operacionais (Plan 1,2 e 3) e uma quarta contendo texto de "ajuda". Far-se-á uma apresentação dessas três planilhas básicas e em seguida será apresentado um exemplo de aplicação das principais funções.

Plan1: Entrada de dados (função: confecciona a tabela e o gráfico automaticamente, na medida em que se digitam os dados)

Nessa planilha, há 40 tabelas de entrada de dados, cada uma com seis colunas. As duas primeiras correspondem à entrada de dados, ou seja, $\mathrm{n}^{0}$ de impactos e profundidade atingida pelo penetrômetro. Na medida em que se insere cada par de dados, o programa calcula automaticamente a camada penetrada (terceira coluna) e, na sequência, a resistência dessa camada em três sistemas de unidade (impactos/dm; kgf/ $\mathrm{cm}^{2} ; \mathrm{MPa}$ ), conforme figura 1 . A cada entrada de dados de campo na Plan1, a tabela de resistência e o respectivo gráfico vão sendo confeccionados automaticamente. E possível utilizar esse programa no campo, com um laptop ou tablete, permitindo conhecer a curva de resistência na medida em que se faz a leitura no campo. Há um comando "Limpar dados de entrada?" utilizado para a entrada de novo conjunto de dados.

\section{Plan2: Equalização da profundidade (função avançada)}

A Plan1, anterior, realiza uma função normalmente esperada pelo usuário: transformar os dados de campo de cada perfil em uma tabela e desenhar o gráfico. Essa Plan2 realiza por meio de uma MACRO em linguagem VBA a equalização, ou seja, a padronização dos intervalos de profundidade (Figura 1). Para utilizá-la, após entrar com os dados de campo na Plan1, o usuário deve abrir a Plan2 e clicar em: "Gerar tabela em camadas de espessura constante".O programa pedirá o valor da espessura da camada, sugerindo como padrão " $5 \mathrm{~cm}$ ". Em seguida, apresentará a opção para selecionar " 1 - Todos os perfis"; "2- Uma sequência de perfis" ou " 3 - Entrada aleatória”. Em continuidade, o programa irá automaticamente coletar os dados da Plan1, promover a equalização e finalmente lançar, na Plan2, a tabela equalizada e em formato científico, apropriado para compor relatórios e artigos.

\section{Plan3 (entrada rápida e tabela de campo)}

Não é uma planilha essencial, apenas facilitadora. Permite a entrada de dados por meio de apenas duas colunas para cada perfil. Após o término da digitação, os dados necessitam ser lançados na Plan1 pelo comando: "Lançar dados desta Plan3 para planilha Plan1". O inverso também é possível: "Lançar dados da Plan1 para Plan3”. Pode ser impressa para anotar dados de campo.

\section{Exemplo de aplicação: computar os dados de campo contidos no quadro 1}

Os dados de campo (Quadro 1) podem entrar no programa pela Plan3 e lançados na Plan1 ou serem digitados diretamente na Plan1, resultando o quadro 2. A Plan1 (Quadro 2) já contém os dados de resistência do solo e os gráficos dos perfis. Note-se, porém, que cada perfil é representado por uma tabela individual. Utilizando a Plan2 (comandos sequenciais: "Gerar tabela em camadas de espessura constante", " $5 \mathrm{~cm}$ " e "1- Todos os perfis"), o programa equaliza a profundidade, unificando, na planilha Plan2, os seis perfis em uma única tabela (Quadro 3). Os dados são referentes a um estudo com cana-de-açúcar com amostragem na linha e na entrelinha de plantio. É possível fazer a separação para analisar (linha $\times$ entrelinha). Linha da cana: Plan2: "Gerar tabela em camadas de espessura constante", "5 cm" e " 3 Entrada aleatória: entrar com os perfis 1, 3 e 5, correspondentes às medidas na linha de cana". Dessa forma, gera-se o quadro 4 (linha). Repetindo-se o procedimento com os perfis 2,4 e 6 , gera-se o quadro 5 (entrelinha). No quadro 6, apresenta-se simplesmente a cópia de colunas das médias das duas tabelas anteriores, comparando diretamente as duas posições.

Conforme já exposto, é possível selecionar o intervalo de profundidade de acordo com a necessidade. Supondo uma pesquisa que relaciona a quantidade de raízes de $0-20$ e $20-40 \mathrm{~cm}$ de profundidade com a resistência do solo, nesse caso o intervalo a ser selecionado é $20 \mathrm{~cm}$. Entretanto, pode haver o interesse de utilizar intervalos menores, como intervalos de 1,5 cm de profundidade. 
Quadro 1. Dados de campo de seis perfis

\begin{tabular}{|c|c|c|c|c|c|c|c|c|c|c|c|}
\hline \multicolumn{2}{|c|}{ Perfil 1} & \multicolumn{2}{|c|}{ Perfil 2} & \multicolumn{2}{|c|}{ Perfil 3} & \multicolumn{2}{|c|}{ Perfil 4} & \multicolumn{2}{|c|}{ Perfil 5} & \multicolumn{2}{|c|}{ Perfil 6} \\
\hline \multicolumn{2}{|c|}{$\begin{array}{l}\text { Linha } \\
\text { da cana }\end{array}$} & \multicolumn{2}{|c|}{$\begin{array}{c}\text { Entrelinha } \\
\text { da cana }\end{array}$} & \multicolumn{2}{|c|}{$\begin{array}{c}\text { Linha } \\
\text { da cana }\end{array}$} & \multicolumn{2}{|c|}{$\begin{array}{c}\text { Entrelinha } \\
\text { da cana }\end{array}$} & \multicolumn{2}{|c|}{$\begin{array}{c}\text { Linha } \\
\text { da cana }\end{array}$} & \multicolumn{2}{|c|}{$\begin{array}{c}\text { Entrelinha } \\
\text { da cana }\end{array}$} \\
\hline Impacto & Prof. & Impacto & Prof. & Impacto & Prof. & Impacto & Prof. & Impact & Prof. & Impacto & Prof. \\
\hline $\mathrm{n}^{\mathrm{o}}$ & $\mathrm{cm}$ & $\mathrm{n}^{\mathrm{o}}$ & $\mathrm{cm}$ & $\mathrm{n}^{\mathrm{o}}$ & $\mathrm{cm}$ & $\mathrm{n}^{\mathrm{o}}$ & $\mathrm{cm}$ & $n^{o}$ & $\mathrm{~cm}$ & $\mathrm{n}^{\mathrm{o}}$ & $\mathrm{cm}$ \\
\hline 0 & 10,5 & 0 & 6,0 & 0 & 12,0 & 0 & 4,0 & 0 & 10,5 & 0 & 6,0 \\
\hline 1 & 13,5 & 2 & 11,0 & 1 & 16,5 & 1 & 11,0 & 1 & 15,0 & 1 & 11,0 \\
\hline 2 & 17,5 & 3 & 16,5 & 2 & 22,0 & 2 & 14,5 & 2 & 20,0 & 3 & 16,5 \\
\hline 2 & 22,0 & 3 & 24,0 & 2 & 28,0 & 3 & 19,0 & 2 & 25,0 & 3 & 20,0 \\
\hline 2 & 28,0 & 1 & 29,0 & 2 & 34,0 & 3 & 25,0 & 2 & 30,0 & 3 & 30,0 \\
\hline 1 & 32,5 & 1 & 34,0 & 1 & 37,5 & 2 & 32,5 & 1 & 33,0 & 1 & 35,0 \\
\hline 1 & 37,5 & 1 & 40,5 & 1 & 42,5 & 1 & 37,0 & 1 & 38,0 & 1 & 40,0 \\
\hline 1 & 43,0 & 1 & 47,0 & 1 & 48,0 & 1 & 43,0 & 1 & 43,0 & 1 & 45,0 \\
\hline 1 & 49,0 & 1 & 53,5 & 1 & 53,0 & 1 & 50,0 & 1 & 49,0 & 1 & 52,0 \\
\hline 1 & 56,5 & 1 & 59,0 & 1 & 58,0 & 1 & 55,5 & 1 & 56,0 & 1 & 58,0 \\
\hline 1 & 66,0 & 1 & 63,0 & 1 & 63,0 & 1 & 60,0 & 1 & 67,0 & 1 & 61,0 \\
\hline
\end{tabular}

Observação: O número de impactos maior, nos casos 2 e 3, é utilizado em camadas de maior resistência, onde ocorre baixa penetração. Esse procedimento tem sido recomendado: (a) para tornar ágil a leitura em camadas muito resistentes e (b) para evitar a ocorrência de um erro relativo de leitura maior. Com a introdução de uma régua milimétrica de leitura (Stolf et al., 2012), essa prática deixa de ser necessária.

Quadro 2. Plan1 (tela do computador). Entrada dos dados de campo para cálculo da resistência. Perfis de 1 a 6 (coube apenas 4). Note-se que para cada perfil é necessária a existência de uma tabela individual (solução na próxima tabela, por meio do uso da Plan2)

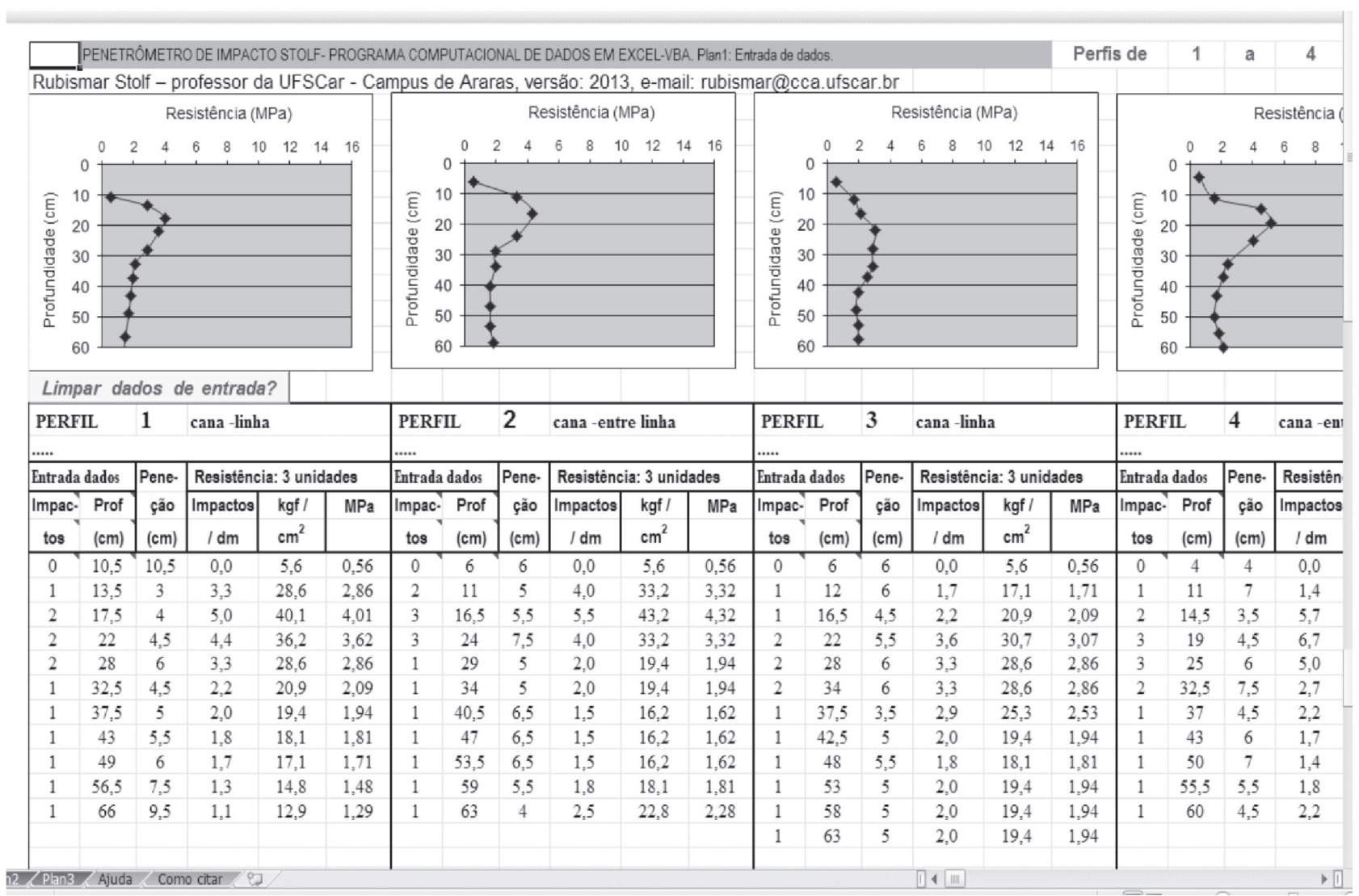


Quadro 3. Plan2. com os seis perfis $(1,2,3,4,5,6)$ equalizados em intervalos de profundidade de 5 cm e condensados em uma única tabela, com a média geral na última coluna com o gráfico

\begin{tabular}{cccccccccc}
\hline \multirow{2}{*}{ Prof. em camada } & Prof. média & \multicolumn{6}{c}{ Resistência } \\
\cline { 5 - 10 } & & $\mathbf{1}$ & $\mathbf{2}$ & $\mathbf{3}$ & $\mathbf{4}$ & $\mathbf{5}$ & $\mathbf{6}$ & Média \\
\hline $\mathrm{cm}$ & $\mathrm{cm}$ & & & \multicolumn{3}{c}{$\mathrm{MPa}$} \\
$0-5$ & 2,50 & 0,56 & 0,56 & 0,56 & 0,76 & 0,56 & 0,56 & 0,59 \\
$5-10$ & 7,50 & 0,56 & 2,76 & 0,56 & 1,54 & 0,56 & 1,66 & 1,28 \\
$10-15$ & 12,50 & 2,97 & 4,12 & 1,48 & 3,97 & 1,94 & 3,84 & 3,05 \\
$15-20$ & 17,50 & 3,81 & 3,62 & 2,77 & 4,92 & 3,32 & 5,82 & 4,04 \\
$20-25$ & 22,50 & 3,16 & 3,04 & 2,94 & 4,01 & 3,32 & 2,63 & 3,18 \\
$25-30$ & 27,50 & 2,55 & 1,94 & 2,86 & 2,40 & 3,32 & 2,63 & 2,61 \\
$30-35$ & 32,50 & 2,01 & 1,87 & 2,79 & 2,24 & 2,49 & 1,94 & 2,23 \\
$35-40$ & 37,50 & 1,88 & 1,62 & 2,23 & 1,86 & 1,94 & 1,94 & 1,91 \\
$40-45$ & 42,50 & 1,77 & 1,62 & 1,88 & 1,64 & 1,85 & 1,94 & 1,78 \\
$45-50$ & 47,50 & 1,66 & 1,62 & 1,86 & 1,54 & 1,68 & 1,54 & 1,65 \\
$50-55$ & 52,50 & 1,48 & 1,68 & 1,94 & 1,81 & 1,54 & 1,64 & 1,68 \\
\hline
\end{tabular}

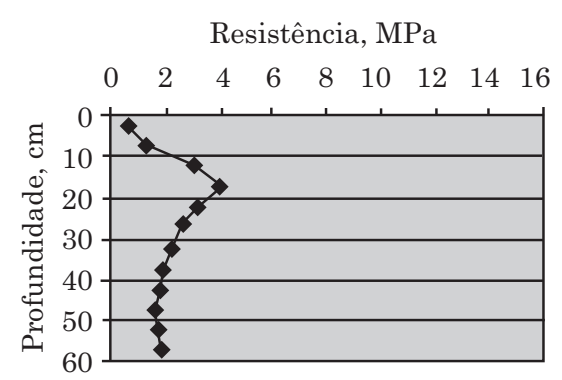

Quadro 4. Plan2. com equalização apenas da linha de cana (perfis 1, 3 e 5)

\begin{tabular}{cccccc}
\hline \multirow{2}{*}{ Prof. em camada } & Prof. média & \multicolumn{4}{c}{ Resistência } \\
\cline { 3 - 6 } & & $\mathbf{1}$ & $\mathbf{3}$ & $\mathbf{5}$ & Média \\
\hline cm & 2,50 & & $\mathrm{MPa}$ & & \\
$0-5$ & 7,50 & 0,56 & 0,56 & 0,56 & 0,56 \\
$5-10$ & 12,50 & 2,56 & 0,56 & 0,56 & 0,56 \\
$10-15$ & 17,50 & 3,81 & 2,77 & 3,32 & 3,30 \\
$15-20$ & 22,50 & 3,16 & 2,94 & 3,32 & 3,14 \\
$20-25$ & 27,50 & 2,55 & 2,86 & 3,32 & 2,91 \\
$25-30$ & 32,50 & 2,01 & 2,79 & 2,49 & 2,43 \\
$30-35$ & 37,50 & 1,88 & 2,23 & 1,94 & 2,02 \\
$35-40$ & 42,50 & 1,77 & 1,88 & 1,85 & 1,83 \\
$40-45$ & 47,50 & 1,66 & 1,86 & 1,68 & 1,73 \\
$45-50$ & 52,50 & 1,48 & 1,94 & 1,54 & 1,65 \\
$50-55$ & 57,50 & 1,34 & 1,94 & 1,26 & 1,51 \\
$55-60$ & & & & & \\
\hline
\end{tabular}

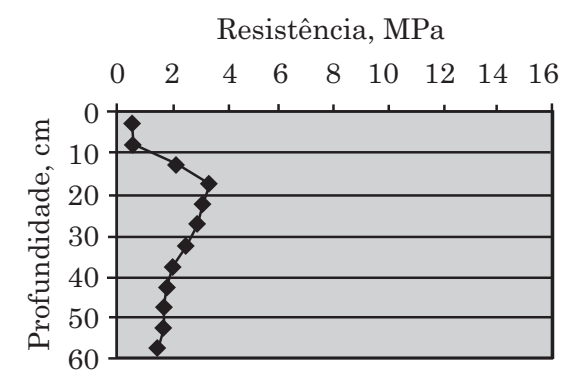

Quadro 5. Plan2. com equalização apenas na entrelinha de cana (perfis 2, 4 e 6)

\begin{tabular}{cccccc}
\hline \multirow{2}{*}{ Prof. em camada } & Prof. média & \multicolumn{4}{c}{ Resistência } \\
\cline { 3 - 6 } & & $\mathbf{2}$ & $\mathbf{4}$ & $\mathbf{6}$ & Média \\
\hline $\mathrm{cm}$ & $\mathrm{cm}$ & & $\mathrm{MPa}$ & & \\
$0-5$ & 2,50 & 0,56 & 0,76 & 0,56 & 0,63 \\
$5-10$ & 7,50 & 2,76 & 1,54 & 1,66 & 1,99 \\
$10-15$ & 12,50 & 4,12 & 3,97 & 3,84 & 3,98 \\
$15-20$ & 17,50 & 3,62 & 4,92 & 5,82 & 4,79 \\
$20-25$ & 22,50 & 3,04 & 4,01 & 2,63 & 3,22 \\
$25-30$ & 27,50 & 1,94 & 2,40 & 2,63 & 2,32 \\
$30-35$ & 32,50 & 1,87 & 2,24 & 1,94 & 2,02 \\
$35-40$ & 37,50 & 1,62 & 1,86 & 1,94 & 1,81 \\
$40-45$ & 42,50 & 1,62 & 1,64 & 1,94 & 1,73 \\
$45-50$ & 47,50 & 1,62 & 1,54 & 1,54 & 1,57 \\
$50-55$ & 52,50 & 1,68 & 1,81 & 1,64 & 1,71 \\
$55-60$ & 57,50 & 1,91 & 2,06 & 2,17 & 2,05 \\
\hline
\end{tabular}

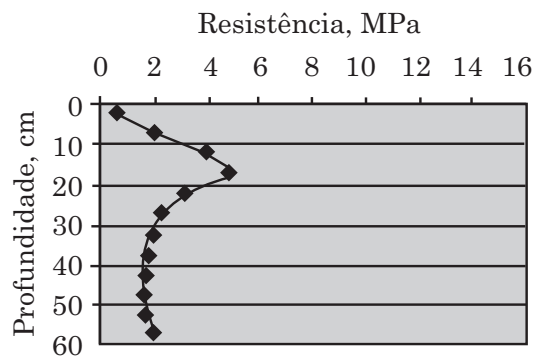


Quadro 6. Comparação: linha $\times$ entrelinha. As colunas foram copiadas e coladas dos quadros 4 e 5

\begin{tabular}{cccc}
\hline Prof. em camada & Prof. média & Linha média & Entrelinha média \\
\hline $\mathrm{cm}$ & $\mathrm{cm}$ & \multicolumn{2}{c}{$\mathrm{MPa}$} \\
$0-5$ & 2,50 & 0,56 & 0,63 \\
$5-10$ & 7,50 & 0,56 & 1,99 \\
$10-15$ & 12,50 & 2,13 & 3,98 \\
$15-20$ & 17,50 & 3,30 & 4,79 \\
$20-25$ & 22,50 & 3,14 & 3,22 \\
$25-30$ & 27,50 & 2,91 & 2,32 \\
$30-35$ & 32,50 & 2,43 & 2,02 \\
$35-40$ & 37,50 & 2,02 & 1,81 \\
$40-45$ & 42,50 & 1,83 & 1,73 \\
$45-50$ & 47,50 & 1,73 & 1,57 \\
$50-55$ & 52,50 & 1,65 & 1,71 \\
$55-60$ & 57,50 & 1,51 & 2,05 \\
\hline
\end{tabular}

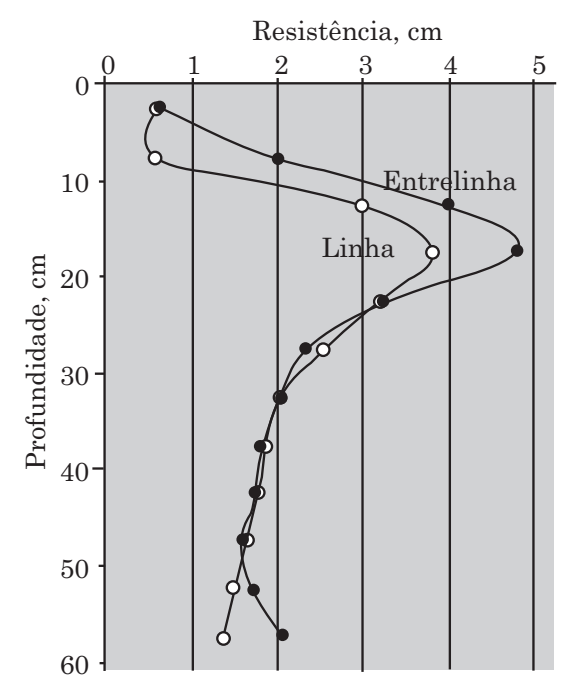

\section{CONCLUSÕES}

Foi possível desenvolver um programa computacional, atingindo os seguintes objetivos:

1. Confeccionar automaticamentea tabela e o gráfico de resistência na medida em que se dá entrada aos dados. Opcionalmente, poder computar no próprio campo para monitorar e visualizar o gráfico de resistência durante a penetração.

2. Desenvolver uma rotina computacional para a equalização da profundidade em camadas constantes, unificando todos os perfis em uma única tabela, obedecendo-se normas do formato científico. Além disso, ao entrar uma série de perfis, o programa permite trabalhar separadamente para efeito de comparação, por exemplo: linha versus entrelinha; mata versus área agrícola; e plantio direto versus convencional.

\section{LITERATURA CITADA}

ALMEIDA, C.X.; CENTURION, J.F.; JORGE, R.F.; BARBOSA, J.C. \& ANDRIOLI, I. Funções de pedotransferência para a curva de resistência do solo à penetração. R. Bras. Ci. Solo, 36:1745-1755, 2012.

ARAUJO, E.A.; LANI, J.L.; AMARAL, E.F. \& GUERRA, A. Uso da terra e propriedades físicas e químicas de Argissolo Amarelo distrófico na Amazônia Ocidental. R. Bras. Ci. Solo, 28:307-315, 2004.

CAMARGO, L.A.; MARQUES JUNIOR, J. \& PEREIRA, G.T. Spatial variability of physical attributes of an Alfisol under different hillslope curvatures. R. Bras. Ci. Solo, 34:617$630,2010$.

CARDOSO, E.L.; SILVA, M.L.N.; CURY, N.; FERREIRA, M.M. \& FRANÇA, D.A.F. Qualidade química e física do solo sob vegetação arbórea nativa e pastagens no Pantanal SulMato-Grossense. R. Bras. Ci. Solo, 35:613-322, 2011.
CARVALHO, A.P.V.; DIAS, H.C.T.; PAIVA, H.N. \& TONELLO, K.C. Resistência mecânica do solo à penetração na bacia hidrográfica do Riacho Fundo, Felixlândia-MG. R. Árvore, 36:1091-1098, 2012.

CASTAGNARA, D.D.; KAWARTTE JUNIOR, N.; ZOZ, T.; PIANO, J.T.; ROSSOL, C.D.; OLIVEIRA, P.S.R. \& SAIDELET, E.P. Atributos físicos deLatossolo Vermelho sob pousio ou cultivado com forrageiras tropicais sob pastejo. Biosci. J., 28:150-158, 2012.

CAVALLINI, M.C.; ANDREOTTI, M.; OLIVEIRA, L.L.; PARIZ, C.M. \& CARVALHO, M.P. Relações entre produtividade de Brachiaria brizantha e atributos físicos de um Latossolo do cerrado. R. Bras. Ci. Solo, 34:1007-1015, 2010.

COBRA, R.L.; PINTO, L.V.A.; SOUZA, R.X.; PEREIRA, M.W.M. \& PEREIRA, A.J. Resistência à penetração do solo de uma encosta: Efeitos de espaçamento de plantio e idade da gramínea Vetiver. R. Agrogeoamb., 4:1-9, 2012.

CORREA, R.S. \& BENTO, M.A.B. Qualidade do substrato minerado de uma área de empréstimo revegetada no Distrito Federal. R. Bras. Ci. Solo, 34:1435-1443, 2010.

CORTEZ, J.W.; ALVES, A.D.S.; MOURA, M.R.D.; OLSZEVSKI, N. \& NAGAHAMA, H.J. Atributos físicos do Argissolo Amarelo do semiárido nordestino sob sistemas de preparo. R. Bras. Ci. Solo, 35:1207-1216, 2011.

CostA, M.S.S.M.; PIVETTA, L.A.; COSTA, L.A.M.; PIVETTA, L.G.; CASTOLDI, G. \& STEINER, F. Atributos físicos do solo e produtividade do milho sob sistemas de manejo e adubações. R. Bras. Eng. Agríc. Amb., 15:810-815, 2011.

CUNHA, E.Q.; STONE, L.F.; FERREIRA, E.P.; DIDONET, A.D. \& MOREIRA, J.A.A. Atributos físicos, químicos e biológicos de solo sob produção orgânica impactados por sistemas de cultivo. R. Bras. Eng. Agríc. Amb., 16:5663, 2012. 
DEBIASI, H. \& FRANCHINI, J.C. Atributos físicos do solo e produtividade da soja em sistema de integração lavourapecuária com braquiária e soja. Ci. Rural, 42:1180-1186, 2012.

ECCO, M.; CARVALHO, L. \& FERRARI, L. Variabilidade espacial da resistência do solo a penetração em área cultivada com cana-de-açúcar na safra 2008/2009. Agrarian, 5:263-269, 2012.

EFFGEN, T.A.M.; PASSOS, R.R.; ANDRADE, F.V.; LIMA, J.S.L.; REIS, E.F. \& BORGES, E.N. Propriedades físicas do solo em função de manejos em lavouras de cafeeiro conilon. R. Ceres, 59:414-421, 2012.

FERREIRA, W.C.; BOTELHO S.A.; DAVIDE A.C.; FARIA, J.M.R. \& FERREIRA, D.F. Regeneração natural como indicador de recuperação de área degradada a jusante da usina hidrelétrica de Camargos, MG. R. Árvore, 34:651660,2010

FREITAS, I.C.; SANTOS, F.C.V.; CUSTÓDIO FILHO, R.O.; SILVA, N.R.A. \& CORRECHE, V. Resistência à penetração em NeossoloQuartzarênico submetido a diferentes formas de manejo. R. Bras. Eng. Agríc. Amb., 16:1275-1281, 2012.

GREGO, C.R.; RODRIGUES, C.A.G.; NOGUEIRA, S.F.; GIMENES, F.M.A.; OLIVEIRA, A.; ALMEIDA, C.G.F.; FURTADO, A.L.S. \& DEMARCHI, J.J.A.A. Variabilidade espacial do solo e da biomassa epígea de pastagem, identificada por meio de geostatística. Pesq. Agropec. Bras., 47:1404-1412, 2012.

GUEDES FILHO, O.; VIEIRA, S.R.; CHIBA, M.K.; NAGUMO, C.H. \& DECHEN, S.C.F. Spatial and temporal variability of crop yield and some Rhodic Hapludox properties under no-tillage. R. Bras. Ci. Solo, 34:1-14, 2010.

HOMMA, S.K. Efeito do manejo alternativo sobre a descompatação do solo, fungos micorrízicosarbusculares nativos e produção em pomar convencional de Targor Murcott. Piracicaba, Universidade de São Paulo, 2005. 101p. (Dissertação de Mestrado)

HOMMA, S.K.; TOKESHI, H.; MENDES, L.W. \& TSAI, S.M. Long-term application of biomass and reduced use of chemicals alleviate soil compaction and improve soil quality. Soil Till. Res., 120:147-153, 2012.

IAREMA, A.A.; FONTE, L.E.F.; FERNANDES, R.B.A.; SHAEFER, C.E.G.R. \& PEREIRA, L.C. Qualidade física e química do solo em áreas de exploração florestal no Mato Grosso. R. Árvore, 35:737-744, 2011.

LIMA, C.G.R.; CARVALHO, M.P.; NARIMATSU, K.C.P.; SILVA, M.G. \& QUEIROZ, H.A. Atributos físico-químicos de um Latossolo do Cerrado brasileiro e sua relação com características dendrométricas do eucalipto. R. Bras. Ci. Solo, 34:163-173, 2010.

MACHADO, W.; TELLES, T.S.; TAVARES FILHO, J.; GUIMARÃES, M.F.; ALVES, G.B. \& BORGES, J.L.B. Physical properties of a Rhodic Haplustox under two sugarcane harvesting systems. R. Bras. Ci. Solo, 34:18031809,2010

MANFRÉ, L.A.; SILVA, A.M. \& URBAN, R.C. Atributos de qualidade de solos sob dois diferentes tipos de manejo no município de Ibiúna/SP, Brazil. Interciencia, 36:757-763, 2011.
MARASCA, I. Variabilidade espacial da resistência do solo à penetração e teor de água em sistema de plantio direto, na cultura da soja. Biosci. J., 7:239-246, 2011.

MARTINS, C.A.S.; PANDOLFI, F.; PASSOS, R.R.; REIS, E.F. \& CABRAL, M.B.G. Avaliação da compactação de um Latossolo Vermelho-Amarelo sob diferentes coberturas vegetais. Biosci. J.,26:79-83, 2010.

MEDEIROS, J.C.; FIGUEIREDO, G.C. \& MAFRA, A.L. Portable penetrometer for agricultural soil: Sensitivity test to identify critical compaction depth. R. Bras. Ci. Solo, $34: 1823-1829,2010$

MESTAS, R.M.; ROQUE, M.W.; MATSURA, E.E.; BIZARY, D.R. \& PAZ, A. Variabilidad espacial de los atributos físicohídricos del suelo y de la productividad del cultivo de fréjol (Phaseolus vulgaris L.) irrigado bajo un sistema de siembra directa. R. Ci. Agrár., 33:307-313, 2010.

MOLIN, J.P.; DIAS, C.T.S. \& CARBONERA, L. Estudos com penetrometria: Novos equipamentos e amostragem correta. R. Bras. Ci. Solo, 36:584-590, 2012.

MONTANARI, R.; ZAMBIANCO, E.C.; CORREAA, A.R.; PELLIN, D.M.P.; CARVALHO, M.P. \& DALCHIAVON, F.C. Atributos físicos de um Latossolo Vermelho correlacionados linear e espacialmente com a consorciação de guandu com milheto. R. Ceres, 59:125-135, 2012.

MOURA, J.B.; MARASCA, I.; MENESES, L.A.S.; PIRES, W.M. \& MEDEIROS, L.C. Resistência a penetração do solo em pastagem cultivada com Brachiaria decumbens sob aplicação de dejetos líquidos suínos e cama de frango. Global Sci. Technol., 5:162-169, 2012.

OLIVEIRA, C.A.; KLIEMANN, H.J.; CORRECHEL, V. \& SANTOS, F.C.V. Avaliação da retenção de sedimentos pela vegetação ripária pela caracterização morfológica e físico-química do solo. R. Bras. Eng. Agríc. Amb., 14:12811287,2010

PEDRON, F.A.; FINK, J.R.; DALMOLIN, R.S.D. \& AZEVEDO, A.C. Morfologia dos contatos entre Solo-Saprolito-Rocha em Neossolos derivados de arenitos da formação caturrita no Rio Grande do Sul. R. Bras. Ci. Solo, 34:1941-1950, 2010 .

PORTuGAL, A.F.; CostA, O.D.V. \& CostA, L.M. Propriedades físicas e químicas do solo em áreas com sistemas produtivos e mata na região da Zona da Mata mineira. R. Bras. Ci. Solo, 34:575-585, 2010.

RAMOS, F.T.; NUNES, M.C.M.; CAMPOS, D.T.S.; RAMOS, D.M. \& MAIA, J.C.S. Atributos físicos e microbiológicos de um Latossolo Vermelho-Amarelo distrófico típico sob cerrado nativo e monocultivo de soja. R. Bras. Agroecol., 6:79-91, 2011.

RAMOS, F.T.; MONAR, Y.C.; NUNES, M.C.M.; CAMPOS, D.T.S. \& RAMOS, D.T. Indicadores de qualidade em um Latossolo Vermelho-Amarelo sob pastagem extensiva no pantanal matogrossense. R. Caatinga, 23:112-120, 2010.

ROBOREDO, D.; MAIA, J.C.S.; OLIVEIRA, O. \& ROQUE, C.G. Uso de dois penetrômetros na avaliação da resistência mecânica de um Latossolo Vermelho distrófico. Eng. Agríc., 30:308-314, 2010. 
ROQUE, A.A.O.; SOUZA, Z.M.; BARBOSA, R.S. \& SOUZA, G.S. Controle de tráfego agrícola e atributos físicos do solo em área cultivada com cana-de-açúcar. Pesq. Agropec. Bras., 45:744-750, 2010

SILVA FILHO, E.P.; COTTAS, L.R. \& MARINI, G.B.S. Avaliação da compactação dos solos em áreas de pastagens e florestas em Porto Velho-Rondônia. B. Geogr., 28:145-155, 2010

SILVA, D.C.; SILVA, M.L.N.; CURY, N.; OLIVEIRA, A.H.; SOUZA, F.S.; MARTINS, S.G. \& MACEDO, R.L.G. Atributos do solo em sistemas agroflorestais, cultivo convencional e floresta nativa. R. Estudos Amb., 13:77-86, 2011.

SILVA, W.M.; CREMON, C.; MAPELI, N.C.; TIEPPO, R.C.; CARVALHO, J.M.; LONGO, L. \& MAGALHÃES, W.A. Emissão de $\mathrm{CO}_{2}$ e potencial de conservação de $\mathrm{C}$ em solo submetido à aplicação de diferentes adubos orgânicos. Agrarian, 3:34-43, 2010.

SILVA, W.R.N.; NUNES, M.C.M.; CALDEIRA, D.A.S.; ARANTES, E.M. \& SOUZA, L.H.C. Resistência à penetração de um Latossolo Vermelho sob cultivo de canade-açúcar em diferentes manejos. R. Agrotecnol., 3:4961, 2012.

SILVEIRA, D.C.; MELO FILHO, J.F.; SACRAMENTO, J.A.A.S. \& SILVEIRA, E.C.P. Relação umidade versus resistência à penetração para um Argissolo Amarelo distrocoeso no Recôncavo da Bahia. R. Bras. Ci. Solo, 34:659-667, 2010.

SIQUEIRA, D.S.; MARQUES, J. \& PEREIRA, G.T. The use of landforms to predict the variability of soil and orange attributes. Geoderma, 155:55-66, 2010.

SOUZA, Z.M.; MARQUES JUNIOR, J. \& PEREIRA, G.T. Geoestatística e atributos do solo em áreas cultivadas com cana-de-açúcar. Ci. Rural, 40:48-56, 2010.

STOLF, R.; FERNANDES, J. \& FURLANI NETO, V.L. Penetrômetro de impacto modelo IAA/Planalsucar-Stolf: recomendação para seu uso. STAB, 1:18-23, 1983. [(Reeditado: Piracicaba, IAA/PLANALSUCAR, 1983. 9p. (Série Penetrômetro de Impacto. Boletim, 1)]

STOLF, R. \& FAGANELLO, B.F. Utilização do penetrômetro de impacto, modelo IAA/Planalsucar-Stolf, na recomendação do preparo do solo na Usina Nossa Senhora Aparecida (Pontal-SP). STAB, 1:11-22, 1983. (Reeditado: Piracicaba, IAA/Planalsucar, 1984. 14p. (Série Penetrômetro de Impacto. Boletim, 3)).

STOLF, R. A compactação do solo e perspectivas de subsolagem em citrus. Laranja, 2:283-308, 1987.

STOLF, R. Fórmulas de transformação dos dados de penetrômetro de impacto em força/unidade de área. In: CONGRESSO BRASILEIRO DE ENGENHARIA AGRÍCOLA, 19., Piracicaba, 1990. Anais... Piracicaba, Sociedade Brasileira de Engenharia Agrícola, 1990a. p.823-836.
STOLF, R. Comparação dos resultados de resistência do solo obtidos com o penetrômetro de impacto e o convencional. In: CONGRESSO BRASILEIRO DE ENGENHARIA AGRÍCOLA, 19., Piracicaba, 1990. Anais... Piracicaba, Sociedade Brasileira de Engenharia Agrícola, 1990b. p.837-850.

STOLF, R. Teoria e teste experimental de fórmulas de transformação dos dados de penetrômetro de impacto em resistência do solo. R. Bras. Ci. Solo, 15:229-235, 1991a.

STOLF, R. Impact penetrometer Stolf model: Data manipulation program version 2.1. Araras, Universidade Federal de São Carlos, 1991b.

STOLF, R.; CASSEL, D.K.; KING, L.D. \& REICHARDT, K. Measuring mechanical impedance in clayey gravelly soils. R. Bras. Ci. Solo, 22:189-196, 1998.

STOLF, R.; REICHARDT, K. \& VAZ, C.P.M. Response to "Comments on 'Simultaneous measurement of soil penetration resistance and water content with a combined penetrometer - TDR moisture probe' and 'A dynamic cone penetrometer for measuring soil penetration resistance"'. Soil Sci. Soc. Am. J., 69:927-929, 2005.

STOLF, R.; MURAKAMI, J.H.; MANIERO, M.A.; SILVA, L.C.F. \& SOARES, M.R. Incorporação de régua para medida de profundidade no projeto do penetrômetro de impacto Stolf. R. Bras. Ci. Solo, 5:1476-1482, 2012.

TAVARES FILHO, J.; BARBOSA, G.M.C. \& RIBON, A.A. Physical properties of dystrophic Red Latosol (Oxisol) under different agricultural uses. R. Bras. Ci. Solo, 34:925933, 2010.

TAVARES FILHO, J.; FELTRAN, C.T.M.; OLIVEIRA, J.F. \& ALMEIDA, E. Modelling of soil penetration resistance for an Oxisol under no-tillage. R. Bras. Ci. Solo, 36:89-95, 2012.

THEODORO, V.C.A.; CASTRO, F.P. \& ABURAYA, F.H.Indicadores ecológicos de sustentabilidade de unidades de produção agrícola do assentamento Facão Cáceres, MT, Brasil. R. Bras. Agroecol., 6:21-33, 2011.

TIEPPO, R.C.; GABRIEL FILHO, A.; SILVA, S.L. \& GNOATTO, E. Desenvolvimento de um penetrômetro manual eletrônico. Acta Sci. Technol., 36:9-15, 2011.

TORRES, J.L.R.; FABIAN, A.J. \& PEREIRA, M.G. Alterações dos atributos físicos de um Latossolo Vermelho submetido a diferentes sistemas de manejo. Ci. Agrotec., 35:437$445,2011$.

WENDLING, B.; VINHAL-FREITAS, I.C.; OLIVEIRA, R.C.; BABATA, M.M. \& BORGES, E.N. Densidade, agregação e porosidade do solo em áreas de conversão do cerrado em floresta de pinus, pastagem e plantio direto. Biosci. J., 28:256-265, 2012. 